\title{
The Bone Microenvironmental Effect in the Dormancy of Cancer
}

\author{
Weiwei Shen, Yujie Niu, Helong Zhang* \\ Department of Oncology, Tangdu Hospital, Fourth Military Medical University, Xi'an, Shaanxi, China \\ Email: ${ }^{*}$ cnxazhl@163.com
}

Received 20 January 2014; revised 15 February 2014; accepted 22 February 2014

Copyright (C) 2014 by authors and Scientific Research Publishing Inc.

This work is licensed under the Creative Commons Attribution International License (CC BY). http://creativecommons.org/licenses/by/4.0/

\section{(c) (i) Open Access}

\begin{abstract}
Tumors have already threatened human life and health for centuries, especially the recurrent of bone metastases is difficult to cure. Relapse can occur years to decades after resection of primary tumor. This phenomenon is common in many clinical cases and animal experimental studies. The effect of traditional radiotherapy and chemotherapy on relapse is very limited, and the patients' prognosis is poor. Because the delayed occurrence of metastases, researchers put forward a new concept "dormancy", including a single dormant tumor cell (growth-arrest) and tumor mass dormancy (equivalence of the proliferation rate and apoptosis rate). It is probable that dormant tumor cells are the resource of relapse and the main reason of chemotherapy resistance. The mechanisms mediated tumor cell dormancy are complex and poorly understood, and bone marrow microenvironment plays an important role in this process. This review focuses on the bone marrow microenvironmental effect in inducing cancer cells to dormancy uncovered by the latest researches.
\end{abstract}

Keywords

Bone Marrow, Tumor, Metastasis, Dormancy

\section{Introduction}

The traditional opinion is that only some specific subtype (metastatic subclone) of tumor cells showing up in advanced cancers has the ability to shed from the primary tumor to distant organs and form metastases [1]. In other words, metastasis represents a late event in the progression of tumors, and the metastases are formed only by a subset of cancer cells within a primary tumor which has the potential to disseminate to and proliferate in the second sites. However, another hypothesis was proposed, Bernards \& Weinberg considered the metastatic capacity was gained at the early stages of cancer [2]. In consistent, the detection of single circulating tumor cells

${ }^{*}$ Corresponding author. 
(CTCs) in peripheral blood of early cancer patients and disseminated tumor cells (DTCs) in bone marrow (BM), supports the theory proposed by Bernards \& Weinberg. Interestingly, DTCs derived from a variety of epithelial tumors seem to have a propensity to home to BM, including tumors which do not commonly form bone metastases, such as colon cancer. This implies that BM might be a reservoir for DTCs from where they may recirculate into circulating system and then colonize in other distant organs. A significant correlation between the existence of DTCs in BM and metastatic occurrence or tumor relapse has been confirmed by several clinical studies in various tumor types [3]. It is noteworthy that tumor recurrence is likely caused by these DTCs in BM, not only including metastases of bone but also other organs, such as, lung and colon. Besides the detection of DTCs in early tumors, it has been shown that DTCs resist to chemotherapy and hormonal therapy [4] [5], and survive in BM for years to decades. So even resect the primary tumors and take the corresponding radiochemotherapy after surgery, these cells can still survive, and be reactivated by some endogenic or ectogenic factors, proliferating into new lesions [6]-[9]. What's more, DTCs and CTCs do not express Ki-67, the proliferation antigen, which suggests they arrest at G0 or are dormant. This could explain, at least in part, their resistance to chemotherapy [5]. Dormancy is related to cytostatic drug resistance and may be a property of minimal residual disease (MRD) and tumor stem cells (TSCs). Therefore, uncovering the mechanisms about dormancy is critical for nominating new drugs to eliminate DTCs, and prevent disease recurrence.

\section{Evidence for the Existence of Dormant DTCs}

Previously, the dormancy of cancer cells seeded in secondary sites has been described as quiescent solitary cells that neither proliferate nor apoptosis [10]-[12]. Later, researchers defined this state as "dormancy", which actually referred to DTCs surviving growth-arrested (G0). DTCs reside in patients' BM or other organs for a long period without any clinical symptoms. Because many traditional anticancer drugs target dividing cells, dormant cancer cells are thought to be resistant to most drugs that ultimately can cause tumor recurrence [13] [14]. In 2007, Aquirre-Ghiso proposed two different states of "cancer dormancy”, tumor-cell dormancy and tumor mass dormancy [15]. Tumor-cell dormancy refers to single non-cycling DTCs, while tumor-mass dormancy describes a stage where equal rates of cell proliferation and cell apoptosis. Dormancy of tumor cells are closely related to chemoradiotherapy resistance and MRDs. Moreover, there is a high probability that dormant DTCs is the resource of tumor stem cells.

BM DTCs can be detected in early tumors, which are speculated to undergo a cellular dormancy after their early dissemination; this period of dormancy could explain why they may still be detected years or decades after the surgery of the primary tumor, in the absence of any metastatic symptoms [7]. Recently, some mathematical models suggested the half-life of some dormant breast cancer cells (BCCs) were more than 12 years [16]. This is consistent with the phenomenon that metastatic recurrence in patients with breast cancer can occur over 10 years after the removal of primary tumor [17]. As CTCs have been detected in breast cancer patients who have no any metastases up to 22 years after primary tumor resection, it can be claimed that so-called "cured" cancer patients might carry dormant tumor cells [18]. Increasing studies suggest that tumor cells disseminated from early lesions undergo a long period of dormancy in the target organs [19] [20]. Furthermore, a variety of solid tumor DTCs tend to engraft in BM, such as, breast cancer, prostate cancer, lung cancer and colon cancer [21]-[23]. Surprisingly, bone is not the common metastatic organ, but DTCs have been detected in BM, which implying that BM act as a reservoir for DTCs. These DTCs can recirculate into blood and form metastasis in lung, liver, brain and so on. Together, DTCs can lead to not only bone metastases, but also metastases in other sites.

However, not all of the patients with DTCs at diagnosis would relapse definitely. Demonstrated by animal models, a significant fraction of DTCs may die or remain dormancy permanently, which will never develop into overt metastases [12]. Besides, Bidard F.C has found that relapse still can occur in the breast cancer patients without BM DTCs. What's worse, their relapse appeared more earlier than those with BM DTCs. So it is speculated that dormancy is not a necessary stage that all DTCs have to go through and a portion of DTCs directly enter mitotic phage, proliferating into overt metastasis [24]. The mechanisms of dormant DTCs to be "re-activated" and later to form a secondary lesion are a function of its complex interaction with the stromal microenvironment at the secondary site. The somatic aberrations and hereditary components may also influence each process.

\section{The Mechanisms of Inducing Tumor Cells to Dormancy}

The mechanisms involved in DTCs dormancy are complex, molecularly, it is proposed that the balance between 
the p38-mediated stress signals and FAK-ERK-mediated mitogenic signals determines the fate of DTCs [15] [25]. By downregulating cyclin D1 and activating the p53 to p21 and/or p16 to Rb pathways, p38 can induce cell cycle arrest [26] [27]. BM microenvironment can maintain the dormant state of tumor cells resided in it [28], and most of BM DTCs are at G0 [10]. During studying melanoma, it was found that tumor cells entered growth arrest in response to environmental stress when activation of p38-mediated stress-response pathways outpace the mitogenic ERK-activity [29]. Only when these two signaling pathways keep a certain balance, can the tumor cells maintain the normal cell cycle. A number of metastasis suppressor genes, including KISS1, CSRP3, Kai1, MKK4, MKK7, MKK6, Nm23 and RKIP suppress tumor cell proliferation by activating p38 signals, inhibiting ERK kinase, or both [30]. On the contrary, u-PAR can increase ERK/p38 ratio, promoting cell division [31] [32]. Besides, the regulation of TGF $\beta 2$ to cell cycle is also through ERK/p38 signal pathway. TGF $\beta 2$ activates p38 pathway, which can induce the expression of DEC2/SHARP1 and p27, suppressing the cyclin-dependent kinase 4 (CDK 4) and leading to dormancy. Conversely, when inhibit the TGF $\beta$ receptor I or interrupt p38 pathway, dormant DTCs can be re-activated [33]. Next, we will further elaborate the achievement in the study of mechanisms about tumor cell dormancy.

Jung, Y noticed that in the murine model of prostate cancer (PCa), the lesions in the forelimb (humeri, radius, and ulna) were fewer than those formed in the hindlimb regions. Then they found the main distinct cytokine was growth arrest specific-6 (GAS6) which levels were significantly higher in the arms versus hindlimb BM. By subcutaneous injection, cancer cells were implanted into immune competent animals and grew significantly better in the $\mathrm{GAS6}^{-/-}$animals than in $\mathrm{GAS6}^{+/+}$wild-type animals. Similar results were observed in osseous environment, the human PC3 cell line was injected into vertebral body transplants (vossicles) and tumors formed in vossicles derived from $\mathrm{GAS6}^{-/-}$animals were greater than those from $\mathrm{GAS6}^{+/+}$animals. So, they proposed that GAS 6 can induce the dormancy of cancer cells [34]. Meanwhile, Shiozawa Y. et al. not only verified that GAS 6 can regulate PCa cell mitotic cycle, and pointed out that GAS 6 was secreted by osteoblasts in the BM niche [35]. The interaction between osteoblasts and DTCs is complex, Rebecca Marlow established a novel co-culture system of breast cancer cells with BM stromal cells types to model the metastatic niche microenvironment in bone of the breast cancer. In vitro, they confirmed the existence of two kinds of niche in bone, one is consisted of BM stromal cells, which promotes DTCs proliferating; the other one contains osteoblasts, which maintains the dormancy of DTCs. However, this growth arrest was reversible and dormant DTCs were able to restore the proliferative activity when transferred into supportive microenvironment. Furthermore, they demonstrated that the dormancy was modulated by the interactions with osteobalsts and mesenchymal cells in BM, rather than the intrinsic properties of cancer cells [36]. In a word, after DTCs settled in BM niches, niche microenvironment regulated their proliferation or dormancy.

Many studies have explored the localization of DTCs in BM, as well as the function of BM microenvironment on DTCs. Recently, PCa cells have been found target HSC niche during bone metastasis in animal experiment [37]. Further studies found that binding with osteoblasts of niche induces the expression of TANK binding kinase 1 (TBK1) in PCa cells. While, TBK1 interacts with mammalian target of rapamycin (mTOR), inhibiting its activity. Rapamycin is an mTOR inhibitor, inducing cell growth arrest of PCa cells and enhances the resistance of PCa cells to chemotherapy [38]. In addition, rapamycin can also upregulate the expression of CD133, a cancer stem cell marker, in gastric, colorectal, and lung cancer cell lines [39]. In a mouse model of acute myeloid leukemia, Hoshii, T indicated that the inhibition of mTOR signaling may play an important role in inducing tumor dormancy [40]. At last, it is noteworthy that the HSC niche is anoxic and DTCs seeded here arrest at G0, which can reduce the metabolic rate. This may enhance their resistance to various inhibitors of energy metabolism and chemoratiotherapy.

Bone morphogenetic protein (BMP), a well known molecule to regulate bone formation, not only has the function of osteogenesis, but also participates in the normal embryonic development and differentiation of multiple organs. In recent years, it is demonstrated that BMP also participate in controlling cell growth, differentiation, migration and apoptosis of a variety of cells, including malignant tumor cells. PCa cells have been demonstrated to enter a reversible senescent state mediated by BMP 7 in BM [41]. Besides in bone, BMP 7 can also induce dormancy of cancer cells in other organs, such as the lung. Coco, as an antagonist of BMP signaling, exerts this function by blocking BMP ligands, enabling DTCs to overcome the inhibitory effect of BMP and to outgrow into overt metastases. Gao H. et al. found that overexpression of Coco turned dormant DTCs into proliferative state. Whereas, the knock down of Coco induced the majority of cancer cells to enter a dormant state. That is to say, Coco can promote the progress of metastatic breast cancer cells though inhibiting the BMP sig- 
naling [42].

Various chemokines involves in regulating dormancy. Except for those elaborated above, coculture of BCCs with bone marrow stromal cells (BMSCs) demonstrated that miRNA could regulate DTCs dormancy, too. When cocultured those two cells, gap junctions mediated by connexin-43 can form, which allowed the exchange of cytosolic molecules between BCCs and BMSCs. Such as, miRNA (including miR-127, -197, -222, and -223) produced by BMSCs can transfer to BCCs and cause cell cycle arrest [43]. Exosomes derived from stroma, KISS1 [44], myosin light chain kinase (ML-7) and MYC inactivation [45] could also be involved in inducing and maintaining the dormancy of cancer cells, suppressing the formation of metastasis. A few studies show that there is a significant positive correlation between T-cell activation and survival of patients, suggesting immune system can inhibit the growth of tumor and control the MRDs in patients with cancer [46] [47]. Among these molecules, we can not distinguish which is the most important one. Due to the heterogeneity of cancer cells and the complexity of this regulatory mechanism, perhaps different cytokines co-regulate the cells fate in different cancers.

\section{The Exit of Dormant Cells to Proliferate}

In the past, it is believed that metastasis is caused by some mutations of original malignant cells in the late stage of cancer, which can proliferate locally and disseminate to distant organs. However, there is a new hypothesis that regards a malignant tumor as a entity just like an organ. It progresses in a dynamic microenvironment which can either promote or suppress cancer grow. The homeostasis of the microenvironment is important to maintain the dormancy state. In particular, the balance between the factors of growth and angiogenesis, on the one hand, and its corresponding antagonism factors, on the other. Spreading of cancer cells may occur long before the symptoms of primary cancer arise, which makes patients already at a subclinical stage. Both primary and secondary tumors are interacting with various cytokines produced by its surrounding microenvironment, resulting in larger tumors suppressing the growth of smaller tumors.

There is plenty of preclinical evidence that the angiogenic switch is a key step for the Therefore, removal of the primary tumor would lose this inhibitory effect. In addition, wound healing process following surgery may also disrupt the homeostasis of metastases microenvironment, accelerating the growth and vascularization of metastases. Thus, surgery may not always be a good therapy method. Maintaining the state of dormancy may prove a better option to control cancers. As mentioned above, the microenvironment can promote or inhibit the formation of metastases, and the growth factors are more easily to be degraded than growth inhibitors. Consequently, the growth factors propagate mostly by diffusion and act on primary tumor, while the inhibitors can permeate into the vessels and reach remote secondary sites, impeding the growth of metastases. Resection of the primary lesion reduces production of growth inhibitors, which would accelerate the progress of metastases. Additionally, the interruption of these growth-mediated factors caused by primary tumor removal would perturb metastatic homeostasis and force the DTCs to adapt to the microenvironment of the second site, leading to, for some patients, the metastases recurrence [48]-[50]. DTCs exit from dormancy and then proliferate into overt metastases [51]. In the BM microenvironment, endothelial progenitor cells (EPCs) secrete some angiogenesis promoters to regulate the formation of neovasculature in the tumor. Meanwhile, cancer cells produce various cytokines, including VEGF, FGF and GM-CSF, to recruit EPCs to form neovasculature. Cancer cells can also induce the expression of the transcription factor Id1 in the EPCs, whose suppression can block EPC mobilization and cause angiogenesis inhibition, impair tumor growth [52]. Furthermore, Cyrus M proposed that thrombospondin-1 (TSP-1) derived from endothelial induces BCCs quiescence. TSP-1 is an endothelium-derived tumor suppressor and stabilizes the microvasculature through inhibiting the mobility and growth of endothelial cells. Blocking TSP-1 could interrupt the microenvironment in endothelial cells and reactivate dormant DTCs to proliferate. Interestingly, sprouting neovasculature is lack of TSP- 1 and rich in pro-tumor factors TGF- $\beta 1$ and POSTN, suggesting that tumors may escape growth inhibition and progress rapidly. They confirmed that stable microvasculature constituted a dormant niche, which maintained DTCs at G0; whereas sprouting endothelial tips promoted its outgrowth [53].

Although, the conditions required for the persistence of dormancy or the escape from the dormant state into the active state of metastasis formation are poorly understood, it is certain that there is a close relationship between this transformation and the microenvironment of BM. As elaborated before, when the p38 signaling pathway predominates in the BM microenvironment, DTCs enter the dormant state. Otherwise, if the ERK pathway predominates, dormant DTCs can be re-activated. The mechanisms involved in tumor cell dormancy 
are complex and consist of intricate molecular regulation network.

\section{Conclusions}

The theory of dormancy provides a new perspective for the treatment of tumor. Future research endeavors targeting this question will probably shed much needed light on the mechanisms of clinical DTCs dormancy and perhaps nominate therapeutic strategies to target MRDs before they grow into overt metastases.

Tessa Holyoake. et al. showed that cultured CD34+ stem cells isolated from BCR-ABL positive chronic myeloid leukemia (CML) patients contain non-cycling cells, and that these are resistant to the tyrosine kinase inhibitor imatinib mesylate (IM) [54]. Generally, most CML patients respond very well to Imatinib. Nevertheless, stopping imatinib treatment often leads to relapse even for patients showing complete responding at first. It is likely that a few dormant CML stem cells which are resistant to imatinib retained in these patients [55]. So, overcoming dormancy is a critical step to cure CML patients, as well as other cancers contained dormant DTCs. The dormancy of tumor cells may be the main mechanism of chemotherapy resistance, because most chemotherapeutic drugs act on the active cells. So traditional chemotherapy rarely has effect on the non-cycling cells. Recent studies have demonstrated that INF $\alpha$, G-CSF and $\mathrm{As}_{2} \mathrm{O}_{3}$ could re-activate dormant tumor cells and restore the sensitivity of chemotherapy. The treatment of INF $\alpha$, G-CSF or $\mathrm{As}_{2} \mathrm{O}_{3}$ followed by traditional chemotherapy can effectively remove the dormant cancer cells [56]. This strategy provides a new treatment for eliminating MRDs.

Because it is the BM niche microenvironment that sustains DTCs dormancy, mobilization of dormant DTCs from this protective site might be a quantum leap in eliminating MRDs and improving the patients' prognosis. CXCL 12/CXCR 4 axis mediates the adhesion of DTCs to BM and it was showed by a study in multiple myeloma that mobilization by Plerixafor resulted in an increased sensitivity of cancer cells to chemotherapy [57] [58]. So, interruption of the adhesive molecule and detachment from their niches can sensitize tumor cells to chemotherapy. Except for CXCL 12/CXCR 4, many other molecules also involve in mediating DTCs to adhere to BM niche, such as, integrin and cadherin, which can also be the target to detach DTCs from BM. In addition, siRNA induces dormant D-HEp3 cells to apoptosis by downregulating ATF $\alpha$ or Rhe, removing the dormant tumor cells [59].

There is another method to treat dormant cells, maintaining its dormancy permanently. A few studies have found that longer disease-free survival periods were associated with B-Raf [60]. Thus, it is possible to keep B-Raf positive residual melanoma cells dormant by treating the patients with B-Raf or Mek1/2 inhibitors during their asymptomatic periods. If this is the case, we can make new drugs to maintain MRDs dormancy by treating patients during asymptomatic conditions.

A significant correlation between the existence of DTCs in BM and metastatic relapse has been verified by various tumor types. And it is important to investigate the mechanistic processes because it is highly likely that dormant DTCs will finally be the source of subsequent overt metastases years to decades after primary tumor resection.

\section{Funding}

National Natural Science Foundation of China (grant nos.: 81172011, 81272348).

\section{References}

[1] Fidler, I.J. and Hart, I.R. (1982) Biological Diversity in Metastatic Neoplasms: Origins and Implications. Science, 217, 998-1003. http://dx.doi.org/10.1126/science.7112116

[2] Bernards, R. and Weinberg, R.A. (2002) A Progression Puzzle. Nature, 418, 823. http://dx.doi.org/10.1038/418823a

[3] Pantel, K. and Brakenhoff, R.H. (2004) Dissecting the Metastatic Cascade. Nature Reviews Cancer, 4, 448-456. http://dx.doi.org/10.1038/nrc1370

[4] Wimberger, P., Heubner, M., Otterbach, F., Fehm, T., Kimmig, R. and Kasimir-Bauer, S. (2007) Influence of Platinum-Based Chemotherapy on Disseminated Tumor Cells in Blood and Bone Marrow of Patients with Ovarian Cancer. Gynecologic Oncology, 107, 331-338. http://dx.doi.org/10.1016/j.ygyno.2007.07.073

[5] Muller, V., Stahmann, N., Riethdorf, S., Rau, T., Zabel, T., Goetz, A., et al. (2005) Circulating Tumor Cells in Breast Cancer: Correlation to Bone Marrow Micrometastases, Heterogeneous Response to Systemic Therapy and Low Proliferative Activity. Clinical Cancer Research, 11, 3678-3685. http://dx.doi.org/10.1158/1078-0432.CCR-04-2469 
[6] Naume, B., Wiedswang, G., Borgen, E., Kvalheim, G., Karesen, R., Qvist, H., et al. (2004) The Prognostic Value of Isolated Tumor Cells in Bone Marrow in Breast Cancer Patients: Evaluation of Morphological Categories and the Number of Clinically Significant Cells. Clinical Cancer Research, 10, 3091-3097. http://dx.doi.org/10.1158/1078-0432.CCR-03-0373

[7] Wiedswang, G., Borgen, E., Karesen, R., Qvist, H., Janbu, J., Kvalheim, G., et al. (2004) Isolated Tumor Cells in Bone Marrow Three Years after Diagnosis in Disease-Free Breast Cancer Patients Predict Unfavorable Clinical Outcome. Clinical Cancer Research, 10, 5342-5348. http://dx.doi.org/10.1158/1078-0432.CCR-04-0245

[8] Janni, W., Rack, B., Schindlbeck, C., Strobl, B., Rjosk, D., Braun, S., et al. (2005) The Persistence of Isolated Tumor Cells in Bone Marrow from Patients with Breast Carcinoma Predicts an Increased Risk for Recurrence. Cancer, 103, 884-891. http://dx.doi.org/10.1002/cncr.20834

[9] Slade, M.J., Singh, A., Smith, B.M., Tripuraneni, G., Hall, E., Peckitt, C., et al. (2005) Persistence of Bone Marrow Micrometastases in Patients Receiving Adjuvant Therapy for Breast Cancer: Results at 4 Years. International Journal of Cancer, 114, 94-100. http://dx.doi.org/10.1002/ijc.20655

[10] Naumov, G.N., Macdonald, I.C., Weinmeister, P.M., Kerkvliet, N., Nadkarni, K.V., Wilson, S.M., et al. (2002) Persistence of Solitary Mammary Carcinoma Cells in a Secondary Site: A Possible Contributor to Dormancy. Cancer Research, 62, 2162-2168.

[11] Townson, J.L. and Chambers, A.F. (2006) Dormancy of Solitary Metastatic Cells. Cell Cycle, 5, 1744-1750. http://dx.doi.org/10.4161/cc.5.16.2864

[12] Chambers, A.F., Groom, A.C. and Macdonald, I.C. (2002) Dissemination and Growth of Cancer Cells in Metastatic Sites. Nature Reviews Cancer, 2, 563-572. http://dx.doi.org/10.1038/nrc865

[13] Vessella, R.L., Pantel, K. and Mohla, S. (2007) Tumor Cell Dormancy: An NCI Workshop Report. Cancer Biology \& Therapy, 6, 1496-1504. http://dx.doi.org/10.4161/cbt.6.9.4828

[14] Quesnel, B. (2008) Dormant Tumor Cells as a Therapeutic Target? Cancer Letters, 267, 10-17. http://dx.doi.org/10.1016/j.canlet.2008.02.055

[15] Aguirre-Ghiso, J.A. (2007) Models, Mechanisms and Clinical Evidence for Cancer Dormancy. Nature Reviews Cancer, 7, 834-846. http://dx.doi.org/10.1038/nrc2256

[16] Willis, L., Alarcon, T., Elia, G., Jones, J.L., Wright, N.A., Tomlinson, I.P., et al. (2010) Breast Cancer Dormancy Can Be Maintained by Small Numbers of Micrometastases. Cancer Research, 70, 4310-4317. http://dx.doi.org/10.1158/0008-5472.CAN-09-3144

[17] Hanrahan, E.O., Gonzalez-Angulo, A.M., Giordano, S.H., Rouzier, R., Broglio, K.R., Hortobagyi, G.N., et al. (2007) Overall Survival and Cause-Specific Mortality of Patients with Stage T1a,bN0M0 Breast Carcinoma. Journal of Clinical Oncology, 25, 4952-4960. http://dx.doi.org/10.1200/JCO.2006.08.0499

[18] Meng, S., Tripathy, D., Frenkel, E.P., Shete, S., Naftalis, E.Z., Huth, J.F., et al. (2004) Circulating Tumor Cells in Patients with Breast Cancer Dormancy. Clinical Cancer Research, 10, 8152-8162. http://dx.doi.org/10.1158/1078-0432.CCR-04-1110

[19] Nguyen, D.X., Bos, P.D. and Massague, J. (2009) Metastasis: From Dissemination to Organ-Specific Colonization. Nature Reviews Cancer, 9, 274-284. http://dx.doi.org/10.1038/nrc2622

[20] Valastyan, S. and Weinberg, R.A. (2011) Tumor Metastasis: Molecular Insights and Evolving Paradigms. Cell, 147, 275-292. http://dx.doi.org/10.1016/j.cell.2011.09.024

[21] Pantel, K., Brakenhoff, R.H. and Brandt, B. (2008) Detection, Clinical Relevance and Specific Biological Properties of Disseminating Tumour Cells. Nature Reviews Cancer, 8, 329-340. http://dx.doi.org/10.1038/nrc2375

[22] Braun, S., Vogl, F.D., Naume, B., Janni, W., Osborne, M.P. and Coombes, R.C., et al. (2005) A Pooled Analysis of Bone Marrow Micrometastasis in Breast Cancer. The New England Journal of Medicine, 353, 793-802. http://dx.doi.org/10.1056/NEJMoa050434

[23] Pantel, K. and Brakenhoff, R.H. (2004) Dissecting the Metastatic Cascade. Nature Reviews Cancer, 4, 448-456. http://dx.doi.org/10.1038/nrc1370

[24] Bidard, F.C., Vincent-Salomon, A., Sigal-Zafrani, B., Rodrigues, M., Dieras, V. and Mignot, L., et al. (2008) Time to Metastatic Relapse and Breast Cancer Cells Dissemination in Bone Marrow at Metastatic Relapse. Clinical \& Experimental Metastasis, 25, 871-887. http://dx.doi.org/10.1007/s10585-008-9203-1

[25] Sosa, M.S., Avivar-Valderas, A., Bragado, P., Wen, H.C. and Aguirre-Ghiso, J.A. (2011) ERK1/2 and p38 $\alpha / \beta$ Signaling in Tumor Cell Quiescence: Opportunities to Control Dormant Residual Disease. Nature Reviews Cancer, 17, 58505857.

[26] Bulavin, D.V., Phillips, C., Nannenga, B., Timofeev, O., Donehower, L.A. and Anderson, C.W., et al. (2004) Inactivation of the Wip1 Phosphatase Inhibits Mammary Tumorigenesis through p38 MAPK-Mediated Activation of the p16 ${ }^{\text {Ink4a }}-$ p19 ${ }^{\text {Arf }}$ Pathway. Nature Genetics, 36, 343-350. http://dx.doi.org/10.1038/ng1317 
[27] Bulavin, D.V., Demidov, O.N., Saito, S., Kauraniemi, P., Phillips, C. and Amundson, S.A., et al. (2002) Amplification of PPM1D in Human Tumors Abrogates p53 Tumor-Suppressor Activity. Nature Genetics, 31, 210-215. http://dx.doi.org/10.1038/ng894

[28] Mahnke, Y.D., Schwendemann, J., Beckhove, P. and Schirrmacher, V. (2005) Maintenance of Long-Term TumourSpecific T-Cell Memory by Residual Dormant Tumour Cells. Immunology, 115, 325-336. http://dx.doi.org/10.1111/j.1365-2567.2005.02163.x.

[29] Ranganathan, A.C., Adam, A.P., Zhang, L. and Aguirre-Ghiso, J.A. (2006) Tumor Cell Dormancy Induced by p38 ${ }^{\text {SAPK }}$ and ER-Stress Signaling: An Adaptive Advantage for Metastatic Cells. Cancer Biology \& Therapy, 5, 729-735. http://dx.doi.org/10.4161/cbt.5.7.2968

[30] Horak, C.E., Lee, J.H., Marshall, J.C., Shreeve, S.M. and Steeg, P.S. (2008) The Role of Metastasis Suppressor Genes in Metastatic Dormancy. APMIS, 116, 586-601. http://dx.doi.org/10.1111/j.1600-0463.2008.01027.x

[31] Aguirre-Ghiso, J.A., Estrada, Y., Liu, D. and Ossowski, L. (2003) ERK (MAPK) Activity as a Determinant of Tumor Growth and Dormancy; Regulation by $\mathrm{p}^{\mathrm{SAPK}}$. Cancer Research, 63, 1684-1695.

[32] Aguirre-Ghiso, J.A., Liu, D., Mignatti, A., Kovalski, K. and Ossowski, L. (2001) Urokinase Receptor and Fibronectin Regulate the ERK ${ }^{\mathrm{MAPK}}$ to $338^{\mathrm{MAPK}}$ Activity Ratios that Determine Carcinoma Cell Proliferation or Dormancy in Vivo. Molecular Biology of the Cell, 12, 863-879. http://dx.doi.org/10.1091/mbc.12.4.863

[33] Bragado, P., Estrada, Y., Parikh, F., Krause, S., Capobianco, C. and Farina, H.G., et al. (2013) TGF-Beta2 Dictates Disseminated Tumour Cell Fate in Target Organs through TGF-Beta-Riii and p38alpha/beta Signalling. Nature Cell Biology, 15, 1351-1361. http://dx.doi.org/10.1038/ncb2861

[34] Jung, Y., Shiozawa, Y., Wang, J., Mcgregor, N., Dai, J. and Park, S.I., et al. (2012) Prevalence of Prostate Cancer Metastases after Intravenous Inoculation Provides Clues into the Molecular Basis of Dormancy in the Bone Marrow Microenvironment. Neoplasia, 14, 429-439.

[35] Shiozawa, Y., Pedersen, E.A., Patel, L.R., Ziegler, A.M., Havens, A.M. and Jung, Y., et al. (2010) GAS6/AXL Axis Regulates Prostate Cancer Invasion, Proliferation, and Survival in the Bone Marrow Niche. Neoplasia, 12, 116-127.

[36] Marlow, R., Honeth, G., Lombardi, S., Cariati, M., Hessey, S. and Pipili, A., et al. (2013) A Novel Model of Dormancy for Bone Metastatic Breast Cancer Cells. Cancer Research, 73, 6886-6899. http://dx.doi.org/10.1158/0008-5472.CAN-13-0991

[37] Shiozawa, Y., Pedersen, E.A., Havens, A.M., Jung, Y., Mishra, A. and Joseph, J., et al. (2011) Human Prostate Cancer Metastases Target the Hematopoietic Stem Cell Niche to Establish Footholds in Mouse Bone Marrow. The Journal of Clinical Investigation, 121, 1298-1312. http://dx.doi.org/10.1172/JCI43414

[38] Kim, J.K., Jung, Y., Wang, J., Joseph, J., Mishra, A. and Hill, E.E., et al. (2013) TBK1 Regulates Prostate Cancer Dormancy through mTORC1 Inhibition. Neoplasia, 15, 1064-1074.

[39] Matsumoto, K., Arao, T., Tanaka, K., Kaneda, H., Kudo, K. and Fujita, Y., et al. (2009) mTORC1 Signal and Hypoxia-Inducible Factor-1 Alpha Regulate CD133 Expression in Cancer Cells. Cancer Research, 69, 7160-7164. http://dx.doi.org/10.1158/0008-5472.CAN-09-1289

[40] Hoshii, T., Tadokoro, Y., Naka, K., Ooshio, T., Muraguchi, T. and Sugiyama, N., et al. (2012) mTORC1 Is Essential for Leukemia Propagation but Not Stem Cell Self-Renewal. The Journal of Clinical Investigation, 122, 2114-2129. http://dx.doi.org/10.1172/JCI62279

[41] Kobayashi, A., Okuda, H., Xing, F., Pandey, P.R., Watabe, M. and Hirota, S., et al. (2011) Bone Morphogenetic Protein 7 in Dormancy and Metastasis of Prostate Cancer Stem-Like Cells in Bone. The Journal of Experimental Medicine, 208, 2641-2655. http://dx.doi.org/10.1084/jem.20110840

[42] Gao, H., Chakraborty, G., Lee-Lim, A.P., Mo, Q., Decker, M. and Vonica, A., et al. (2012) The BMP Inhibitor Coco Reactivates Breast Cancer Cells at Lung Metastatic Sites. Cell, 150, 764-779. http://dx.doi.org/10.1016/j.cell.2012.06.035

[43] Lim, P.K., Bliss, S.A., Patel, S.A., Taborga, M., Dave, M.A. and Gregory, L.A., et al. (2011) Gap Junction-Mediated Import of Microrna from Bone Marrow Stromal Cells Can Elicit Cell Cycle Quiescence in Breast Cancer Cells. Cancer Research, 71, 1550-1560. http://dx.doi.org/10.1158/0008-5472.CAN-10-2372

[44] Nash, K.T., Phadke, P.A., Navenot, J.M., Hurst, D.R., Accavitti-Loper, M.A. and Sztul, E., et al. (2007) Requirement of KISS1 Secretion for Multiple Organ Metastasis Suppression and Maintenance of Tumor Dormancy. The Journal of the National Cancer Institute, 99, 309-321. http://dx.doi.org/10.1093/jnci/djk053

[45] Shachaf, C.M., Kopelman, A.M., Arvanitis, C., Karlsson, A., Beer, S. and Mandl, S., et al. (2004) MYC Inactivation Uncovers Pluripotent Differentiation and Tumour Dormancy in Hepatocellular Cancer. Nature, 431, 1112-1117. http://dx.doi.org/10.1038/nature03043

[46] Koebel, C.M., Vermi, W., Swann, J.B., Zerafa, N., Rodig, S.J. and Old, L.J., et al. (2007) Adaptive Immunity Maintains Occult Cancer in an Equilibrium State. Nature, 450, 903-907. http://dx.doi.org/10.1038/nature06309 
[47] Galon, J., Costes, A., Sanchez-Cabo, F., Kirilovsky, A., Mlecnik, B. and Lagorce-Pages, C., et al. (2006) Type, Density, and Location of Immune Cells within Human Colorectal Tumors Predict Clinical Outcome. Science, 313, 1960 1964. http://dx.doi.org/10.1126/science.1129139

[48] Demicheli, R., Retsky, M.W., Hrushesky, W.J., Baum, M. and Gukas, I.D. (2008) The Effects of Surgery on Tumor Growth: A Century of Investigations. Annals of Oncology, 19, 1821-1828. http://dx.doi.org/10.1093/annonc/mdn386

[49] Retsky, M., Demicheli, R., Hrushesky, W., Baum, M. and Gukas, I. (2010) Surgery Triggers Outgrowth of Latent Distant Disease in Breast Cancer: An Inconvenient Truth? Cancers (Basel), 2, 305-337. http://dx.doi.org/10.3390/cancers2020305

[50] Demicheli, R., Retsky, M.W., Hrushesky, W.J. and Baum, M. (2007) Tumor Dormancy and Surgery-Driven Interruption of Dormancy in Breast Cancer: Learning from Failures. Nature Clinical Practice Oncology, 4, 699-710. http://dx.doi.org/10.1038/ncponc0999

[51] Steeg, P.S. (2006) Tumor Metastasis: Mechanistic Insights and Clinical Challenges. Nature Medicine, 12, 895-904. http://dx.doi.org/10.1038/nm1469

[52] Gao, D., Nolan, D.J., Mellick, A.S., Bambino, K., Mcdonnell, K. and Mittal, V. (2008) Endothelial Progenitor Cells Control the Angiogenic Switch in Mouse Lung Metastasis. Science, 319, 195-198. http://dx.doi.org/10.1126/science.1150224

[53] Ghajar, C.M., Peinado, H., Mori, H., Matei, I.R., Evason, K.J. and Brazier, H., et al. (2013) The Perivascular Niche Regulates Breast Tumour Dormancy. Nature Cell Biology, 15, 807-817. http://dx.doi.org/10.1038/ncb2767

[54] Goldman, J.M., Green, A.R., Holyoake, T., Jamieson, C., Mesa, R. and Mughal, T., et al. (2009) Chronic Myeloproliferative Diseases with and without the Ph Chromosome: Some Unresolved Issues. Leukemia, 23, 1708-1715. http://dx.doi.org/10.1038/leu.2009.142

[55] Goldman, J.M. (2009) Treatment Strategies for CML. Best Practice \& Research Clinical Haematology, 22, $303-313$. http://dx.doi.org/10.1016/j.beha.2009.08.001

[56] Essers, M.A. and Trumpp, A. (2010) Targeting Leukemic Stem Cells by Breaking Their Dormancy. Molecular Oncology, 4, 443-450. http://dx.doi.org/10.1016/j.molonc.2010.06.001

[57] Azab, A.K., Runnels, J.M., Pitsillides, C., Moreau, A.S., Azab, F. and Leleu. X., et al. (2009) CXCR4 Inhibitor AMD3100 Disrupts the Interaction of Multiple Myeloma Cells with the Bone Marrow Microenvironment and Enhances Their Sensitivity to Therapy. Blood, 113, 4341-4351. http://dx.doi.org/10.1182/blood-2008-10-186668

[58] Weisberg, E., Azab, A.K., Manley, P.W., Kung, A.L., Christie, A.L. and Bronson. R., et al. (2012) Inhibition of CXCR4 in CML Cells Disrupts Their Interaction with the Bone Marrow Microenvironment and Sensitizes Them to Nilotinib. Leukemia, 26, 985-990. http://dx.doi.org/10.1038/leu.2011.360

[59] Schewe, D.M. and Aguirre-Ghiso, J.A. (2008) ATF6alpha-Rheb-mTOR Signaling Promotes Survival of Dormant Tumor Cells in Vivo. Proceedings of the National Academy of Sciences of the United States of America, 105, 1051910524. http://dx.doi.org/10.1073/pnas.0800939105

[60] Ossowski, L. and Aguirre-Ghiso, J.A. (2010) Dormancy of Metastatic Melanoma. Pigment Cell \& Melanoma Research, 23, 41-56. http://dx.doi.org/10.1111/j.1755-148X.2009.00647.x 